\title{
Faire valoir ses droits à l'Aide complémentaire santé : les résultats d'une expérimentation sociale
}

\author{
Sophie Guthmuller, Florence Jusot, Jérôme Wittwer et Caroline Desprès *
}

\begin{abstract}
L'Aide complémentaire santé (ACS) ou « chèque santé » a été mise en place en 2005 pour inciter les ménages dont le niveau de vie se situe juste au-dessus du plafond CMU-C à acquérir une couverture complémentaire santé grâce à une subvention. Même si le nombre de bénéficiaires a lentement progressé depuis son introduction, le recours à l'ACS reste faible. Deux hypothèses peuvent expliquer cet état de fait : (1) le défaut d'information sur l'existence du dispositif, son fonctionnement et sur les démarches à entreprendre pour en bénéficier ; (2) un montant d'aide insuffisant, la complémentaire resterait trop chère même après déduction de l'aide.
\end{abstract}

Afin de tester la validité de ces deux hypothèses, une expérimentation sociale contrôlée a été mise en place par l'université Paris-Dauphine à Lille auprès d'un échantillon de 4209 assurés sociaux potentiellement éligibles à l'ACS. Un montant majoré d'aide ainsi qu'un accès différencié à de l'information sur le dispositif ont été proposés de manière aléatoire à certains assurés.

Les résultats montrent de manière robuste que la majoration du montant du « chèque santé » améliore légèrement le taux de recours à l'ACS et permet de mieux cibler les personnes effectivement éligibles. Toutefois, l'ACS reste un dispositif compliqué qui touche difficilement sa cible : au total, seuls $17 \%$ des assurés ont fait une demande d'ACS. Seuls $9 \%$ des assurés invités à participer à une réunion d'information y ont effectivement assisté, et l'invitation à cette réunion a largement découragé les autres assurés, annulant ainsi l'effet de la majoration du chèque. Enfin, seuls $55 \%$ des assurés ayant déposé une demande ont reçu l'aide, les autres s'étant vu refuser l'aide en raison, le plus souvent, de ressources trop élevées. La difficulté à cibler la population éligible et l'incertitude importante sur l'éligibilité qui en résulte sont certainement des freins s'ajoutant à la complexité des démarches.

\footnotetext{
* Sophie Guthmuller: PSL, Université Paris-Dauphine, LEDa-LEGOS, Paris, France.

Florence Jusot: PSL, Université Paris-Dauphine, LEDa-LEGOS.

Jérôme Wittwer : Université Bordeaux Segalen, ISPED et PSL, Université Paris-Dauphine, LEDa-LEGOS.

Caroline Desprès : Institut de recherche et de documentation en économie de la santé (IRDES).

Les auteurs remercient tout particulièrement la Caisse primaire d'assurance maladie de Lille-Douai, la Caisse d'allocations familiales de

Lille, la Caisse régionale d'assurance maladie Nord-Picardie, l'Institut de recherche et de documentation en économie de la santé, l'Institut national d'études démographiques sans qui cet article n'aurait pas vu le jour, Thomas Renaud, pour son travail sur les données d'enquêtes, pour ses remarques et suggestions. Les auteurs restent seuls responsables des erreurs éventuelles qui pourraient subsister dans cet article.

La réalisation de cet article a bénéficié d'un financement du Haut commissariat aux solidarités actives contre la pauvreté (Ministère de la jeunesse et des solidarités actives) dans le cadre de l'appel à projets d'expérimentations sociales 2008 et du soutien de la Chaire Santé, placée sous l'égide de la Fondation du risque (FDR) en partenariat avec PSL, Université Paris-Dauphine, l'Ensae et la MGEN. Sophie Guthmuller remercie la Caisse nationale d'assurance maladie des travailleurs salariés (CNAMTS) pour son financement de thèse.
} 
$\mathbf{E}$ n France, la Sécurité sociale laisse une partie du coût des soins à la charge du patient (ticket modérateur, participation forfaitaire et franchise médicale, dépassement d'honoraire $)^{1}$. Pour se couvrir contre le risque financier résiduel, les ménages peuvent recourir à des contrats d'assurance complémentaire santé prenant en charge tout ou partie du coût laissé à la charge du patient. Dans une telle organisation, le risque de renoncement aux soins pour raisons financières des personnes non couvertes, ou mal couvertes, par une assurance complémentaire santé est réel (Kambia-Chopin et al., 2008; Jusot et Wittwer, 2009 ; Boisguérin et al., 2010 ; Perronnin et al., 2011). Afin de limiter les barrières financières à l'accès aux soins, deux dispositifs ont été introduits : la prise en charge à $100 \%$ par l'Assurance maladie des dépenses de soins afférentes à une affection de longue durée $(\mathrm{ALD})^{2}$, et la Couverture maladie universelle complémentaire (CMU-C) qui offre gratuitement aux ménages les plus pauvres une couverture complémentaire santé (voir encadré 1).

1. Selon les comptes de la santé 2010, l'assurance maladie finance $75,8 \%$ des dépenses de soins. En Europe, d'autres pays (Grande-Bretagne, Pays-Bas, Espagne par exemple) ont opté pour la gratuité des soins (hors franchises non assurables dont le cumul est plafonné et dont les plus pauvres sont affranchis) mais pour un panier de soins plus strictement défini que le panier donnant lieu à remboursement en France.

2. Au 31 décembre 2008, 8,3 millions d'assurés sociaux du régime général de l'Assurance maladie étaient reconnus en ALD, soit près d'un assuré social sur 7 et leurs dépenses représentaient près des 2/3 des dépenses de soins remboursées par l'Assurance maladie (Paita et Weill, 2009). L'exonération du ticket modérateur n'exclut pas que ces patients doivent faire face à des restes à charge relativement importants, en raison, outre les dépenses de santé liées à d'autres maladies, de la participation forfaitaire, de la franchise médicale et des dépassements d'honoraires relatifs aux dépenses afférentes à la maladie prise en charge en ALD (Elbaum, 2008).

\section{Encadré 1}

\section{LES DISPOSITIFS CMU-C ET ACS}

- La CMU-C (Couverture Maladie Universelle Complémentaire) a été instaurée par la loi du 27 juillet 1999. Elle donne accès de droit à une couverture complémentaire gratuite aux individus des ménages les plus pauvres. Au $1^{\mathrm{er}}$ janvier 2009, les ménages dont le niveau de vie pour une personne seule était inférieur à $7447 €$ par an en métropole étaient éligibles (le plafond est

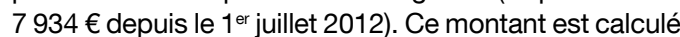
sur la base des revenus des douze mois précédant la demande. Au 31 décembre 2011, 4414347 personnes bénéficiaient de ce dispositif (Fonds CMU, 2012a).

Après instruction de la demande par la CPAM (Caisse primaire d'assurance maladie) compétente, la CMU-C peut-être directement souscrite auprès de la CPAM ou auprès d'un organisme de complémentaire santé. Les prestations offertes équivalent à celles d'un contrat de qualité « moyenne » : les tickets modérateurs sont couverts et les tarifs conventionnels sont opposables pour les consultations chez les généralistes et les spécialistes appliquant des dépassements d'honoraires, les prothèses dentaires et les frais d'optique... La demande doit être renouvelée chaque année.

- L'Aide complémentaire santé (ACS) a été instaurée par la loi du 13 août 2004. Au $1^{\text {er }}$ janvier 2009, elle concernait les ménages dont le niveau de vie annuel est situé entre 7447 et $8936 €$, soit entre le plafond de la $\mathrm{CMU}-\mathrm{C}$ et ce plafond majoré de $20 \%$. Ce niveau de vie est calculé sur la base des revenus des douze mois précédant la demande. Le plafond maximal de ressources donnant droit à l'ACS a beaucoup augmenté depuis son instauration. La majoration du plafond de la CMU-C était de $15 \%$ jusqu'au $1^{\text {er }}$ janvier 2007, $26 \%$ depuis le

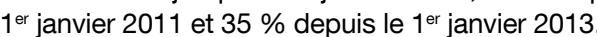

Après instruction de la demande par la CPAM compétente, un ménage éligible se voit délivrer un " chèque santé » (sous forme d'avoir ou de voucher dans la terminologie anglo-saxonne) qu'il peut faire valoir auprès d'un organisme de complémentaire santé (quel qu'il soit) au moment de l'achat d'un contrat dès lors que le contrat est souscrit à titre individuel (les individus bénéficiant d'un contrat à titre collectif, par l'intermédiaire de leur employeur, ne sont pas éligibles à l'ACS). La demande doit être renouvelée chaque année.

Le montant des chèques varie en fonction de l'âge du bénéficiaire. Ils ont été sensiblement augmentés et les tranches d'âge redessinées au $1^{\text {er }}$ août 2009. $\mathrm{Ci}$-dessous, les barèmes en vigueur depuis le $1^{\mathrm{er}}$ janvier 2010 ainsi que ceux en vigueur au $1^{\text {er }}$ janvier 2009, c'est-à-dire au cours de la période durant laquelle l'expérimentation a été conduite. A titre indicatif, le Fonds CMU établit le prix annuel moyen des contrats souscrits par les bénéficiaires de l'ACS (ceux ayant fait valoir leur chèque santé) à $764 €$ en 2009 (Fonds CMU, 2010).

Tableau

Le montant de l'Aide complémentaire santé

\begin{tabular}{|l|c|c|}
\hline \multicolumn{1}{|c|}{ Par personne de } & Montants en vigueur en 2009 & Montants en vigueur depuis le $1^{\text {er janvier 2010 }}$ \\
\hline Moins de 16 ans & 100 & 100 \\
\hline $16-49$ ans & 200 & 200 \\
\hline $50-59$ ans & 400 & 350 \\
\hline 60 ans et plus & & 500 \\
\hline
\end{tabular}

Lecture : les montants d'ACS en vigueur avant le $1^{\text {er }}$ janvier 2009 étaient de $100 €$ par membre du ménage de moins de 25 ans, de $200 €$ par personne âgée entre 25 et 59 ans et de $400 €$ pour chaque personne de 60 ans et plus.

Champ : France entière.

Source : Fonds CMU.

(Pour plus de détails, cf. site du Fonds CMU, www.cmu.fr) 
La question des ménages aux revenus modestes, mais dont le niveau de vie se situe au-delà du plafond CMU-C, s'est rapidement posée. Afin de les inciter à acquérir une couverture complémentaire santé de bonne qualité, mais aussi pour compenser financièrement ceux en détenant déjà une, le dispositif de l'Aide complémentaire santé (ACS) a été mis en place au $1^{\text {er }}$ janvier 2005 (loi du 13 août 2004) et concerne, en janvier 2009, les ménages dont le niveau de vie est situé entre le plafond CMU-C et ce plafond majoré de $20 \%$. En pratique, ces ménages peuvent faire valoir leur droit auprès de leur Caisse primaire d'assurance maladie (CPAM) et bénéficier d'un chèque santé (cf. encadré 1). Présenté à l'organisme de complémentaire santé sollicité, ce chèque donne droit à une réduction sur le prix du contrat dès lors que celui-ci est souscrit à titre individuel et non par l'intermédiaire de l'employeur.

Ce dispositif peut se comprendre comme un instrument de lissage du dispositif d'aide publique à l'accès à une complémentaire santé qui était marqué avant la mise en œuvre de l'ACS par l'effet de seuil généré par le plafond de la CMU-C. À la création du dispositif, la population ciblée était évaluée à 2 millions d'individus (voir encadré 2). Le nombre de bénéficiaires

Encadré 2

\section{LES EFFECTIFS ÉLIGIBLES À L'ACS ET LES BÉNÉFICIAIRES}

Lors de son instauration par la loi du 13 août 2004, l'aide à l'acquisition d'un contrat de couverture complémentaire concernait les ménages dont les revenus se situaient entre le plafond de la CMU-C et ce plafond majoré de $15 \%$. Selon le rapport du Haut conseil à l'avenir de l'Assurance maladie (HCAAM, 2005, p. 64), 2 millions de personnes étaient dans le champ des ressources éligibles en 2005. Le champ des personnes éligibles a été étendu en 2006 dans le cadre du projet de Loi de financement de la Sécurité sociale de 2007 aux ménages dont les revenus se situaient entre le plafond majoré de la $\mathrm{CMU}-\mathrm{C}$ et ce plafond majoré de $20 \%$. L'extension devait correspondre à 650000 personnes éligibles supplémentaires selon le rapport du HCAAM (HCAAM, 2006, p. 38), la Commission des comptes de la sécurité sociale du 26 septembre 2006 prévoyant jusqu'à 2,9 millions de personnes éligibles au dispositif étendu. Cependant, le nombre d'éligibles initialement évoqué était largement surestimé puisque seules sont concernées les personnes non couvertes dans le cadre d'un contrat collectif par leur employeur ou celui d'un membre de leur famille. Or, on peut estimer que $15 \%$ des individus éligibles à l'ACS sont couverts par un contrat collectif si l'on approxime la population éligible à l'ACS par la population non couverte par la CMU-C appartenant au premier décile de niveau de vie, cette estimation passant à $23 \%$ si la population éligible est approximée par le deuxième décile de niveau de vie (Arnould et Vidal, 2008). Ainsi, le HCAAM révisait en 2007 la cible potentielle à 2,2 millions de personnes (HCAAM, 2007, p. 41). Enfin, selon le modèle de microsimulation Ines (INSEE-Études sociales), 1,7 millions de foyers et 3,4 millions de personnes seraient éligibles à l'ACS en 2011, conduisant ainsi à un taux de non recours de 78 \% (Fonds CMU, 2012a).

\section{Graphique \\ Nombre de bénéficiaires ayant utilisé leur attestation}

Nombre de bénéficiaires ayant utilisé leur attestation

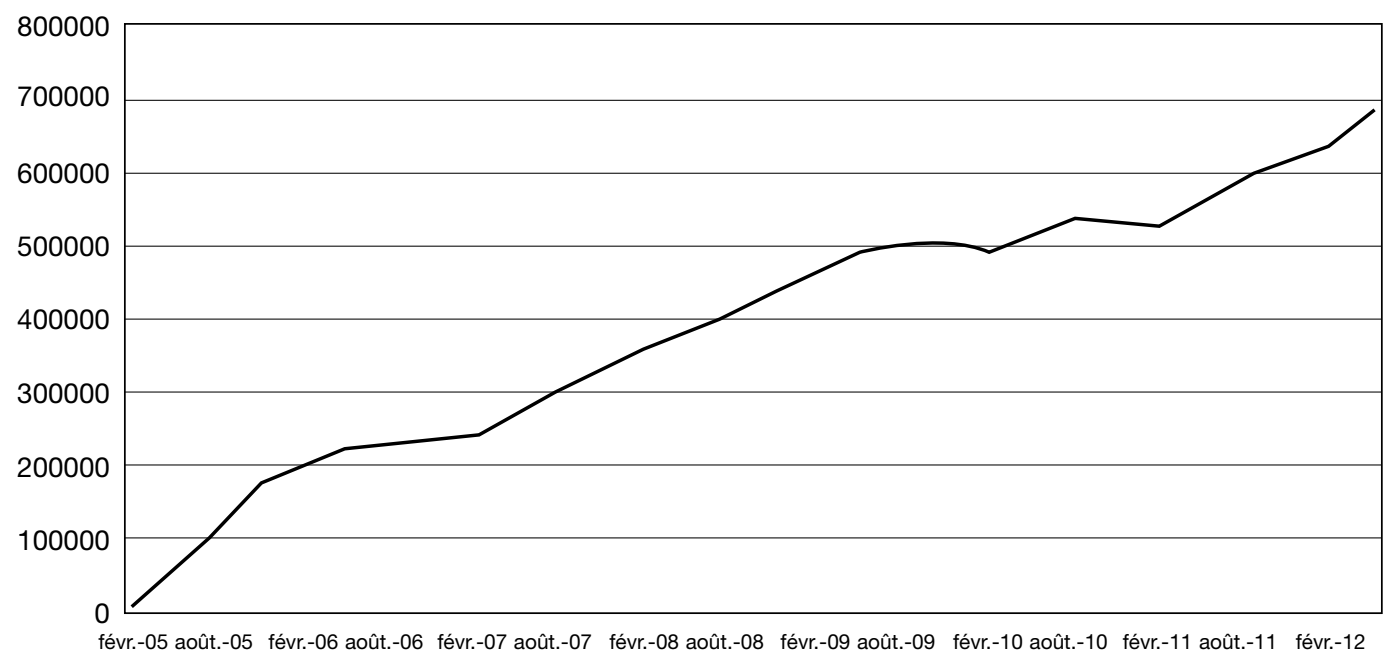

Lecture : en France, en août 2005, 113731 bénéficiaires de l'ACS avaient utilisé leur attestation.

Champ : France métropolitaine.

Source : graphique des auteurs à partir des données du Fonds CMU, juillet 2012 (Fonds CMU, 2012b). 
effectifs de l'ACS a lentement progressé depuis sa mise en place (voir graphique dans l'encadré 2). Cependant, le recours à l'ACS reste faible. Fin 2008, 596626 personnes s'étaient vu délivrer une attestation par les CPAM et seuls 441948 bénéficiaires avaient effectivement utilisé leur attestation auprès d'un organisme complémentaire (Fonds CMU, 2009). Fin 2011, 763079 personnes étaient bénéficiaires d'une attestation et 619957 l'avaient effectivement utilisées (Fonds CMU, 2012a). Cette réalité est d'autant plus surprenante qu'une partie importante des éligibles est couverte par une complémentaire santé souscrite à titre individuel ${ }^{3}$ et qu'à ce titre ils pourraient bénéficier de droit d'une réduction du coût de leur contrat.

Deux grandes hypothèses peuvent être formulées pour expliquer cet état de fait.

La première renvoie au défaut d'information sur le dispositif, tant sur l'existence même du dispositif que sur son principe de fonctionnement ou sur les démarches à entreprendre pour en bénéficier. Il s'agit de la principale hypothèse expliquant le non recours des personnes couvertes par une complémentaire à titre individuel et ne faisant pas valoir leur droit. La campagne d'information par courrier lancée par les CPAM en 2008-2009 avait pour objectif de pallier ce déficit d'information présupposé 4 .

Selon une deuxième hypothèse, le montant $\mathrm{du}$ chèque santé serait insuffisant: une fois déduit ce montant, l'achat d'une complémentaire santé laisserait un reste à payer trop important pour de nombreux ménages. Cette hypothèse s'applique cette fois plus naturellement aux éligibles non couverts par une complémentaire santés. En effet, selon les estimations du fonds CMU, le chèque santé couvrait $50 \%$ du prix des contrats de complémentaire santé en moyenne (Fonds CMU, 2008) ; ces primes pouvant par ailleurs représenter, avant déduction du chèque santé, près de 8 à $10 \%$ du revenu disponible des ménages les plus modestes (Grignon, KambiaChopin, 2009; Jusot et al., 2012 ; Perronnin et al., 2011). Ainsi, c'est sur la base de cette seconde hypothèse qu'une légère revalorisation du chèque santé a été mise en place au $1^{\text {er }}$ janvier 2010 (voir tableau dans l'encadré 1).

L'obstacle de la stigmatisation, entendu au sens large, est également couramment avancé pour expliquer le non recours aux prestations publiques sous conditions de ressources (Hernanz et al., 2004). Le fait de devoir révéler ses ressources à l'administration de la Sécurité sociale mais aussi aux acteurs du système de santé constitueraient un frein au recours. Sur ce dernier point, l'ACS est indiscutablement moins stigmatisante que la CMU-C puisque le seul acteur informé, hors administrations de Sécurité sociale, est l'organisme de complémentaire santé sollicité (Desprès, 2010); le bénéfice de l'ACS n'est pas connu des médecins. Sachant, en outre, que le non recours à la CMU-C est faible comparativement à celui de l'ACS, on est tenté de conclure que l'obstacle de la stigmatisation est un obstacle résiduel pour l'ACS. Il faut cependant rester prudent, d'une part, parce que le bénéfice tiré de l'ACS est moindre que celui tiré de la CMU-C, et, d'autre part, parce que les individus éligibles sont pour la majorité d'entre eux détenteurs d'un contrat de complémentaire santé donc, de ce fait, parfois engagés dans une relation sur le moyen ou long terme avec leur organisme de complémentaire santé.

Quoiqu'il en soit, nous focalisons notre attention, ici, sur les deux explications jugées a priori comme centrales pour expliquer le faible recours à l'ACS : le défaut d'information de la population éligible et l'insuffisance du montant du chèque santé. Pour tester la validité de ces deux hypothèses et estimer rigoureusement les effets à attendre d'une modification de ce dispositif, une expérimentation sociale a été élaborée et mise en œuvre par l'Université Paris-Dauphine en janvier 2009 auprès de 4209 assurés sociaux de la CPAM de Lille-Douai, potentiellement éligibles à l'ACS au regard de leurs ressources ayant donné droit à prestations de la Caisse d'allocations familiales (CAF) de Lille et n'ayant pas fait valoir leur droit à l'ACS à cette date. Nous revenons dans la section suivante sur les questions de méthodes soulevées par l'étude du non recours. Nous présentons ensuite l'expérimentation et notre démarche. Nous détaillerons enfin les résultats avant d'apporter des éléments de conclusion.

3. Avec une approximation de la population éligible à l'ACS par la population non couverte par la CMU-C appartenant au premier décile de niveau de vie, il vient que $53 \%$ des éligibles sont couverts par une complémentaire santé à titre individuel, l'estimation passant à $60 \%$ si l'on approxime la population éligible à l'ACS aux personnes appartenant au deuxième décile de niveau de vie (Arnould et Vidal, 2008).

4. Voir l'encadré 3 pour plus de précisions sur cette campagne d'information.

5. Elle peut néanmoins s'étendre aux individus détenteurs d'une complémentaire santé en partant du principe que les démarches à entreprendre constituent un coût qui doit être couvert par le bénéfice monétaire du chèque. 


\section{Questions de méthode}

É tudier le non recours à une prestation sous condition de ressources, c'est se heurter à deux écueils.

Un écueil est, en premier lieu, celui de la constitution d'un échantillon d'individus ou de ménages éligibles. Cette difficulté est particulièrement sévère dans le cas de l'ACS compte tenu de l'étroitesse de la cible visée par la prestation. Les données d'enquêtes en population générale ne peuvent fournir d'échantillons de taille suffisante. En outre, l'identification de la population éligible est rendue très ardue en raison de la richesse et de la précision de l'information à recueillir pour mettre en œuvre les critères d'éligibilité présidant à l'examen des dossiers par les administrations compétentes ${ }^{6}$. Cette difficulté d'identification rend très difficile la réalisation d'une large enquête représentative de la population éligible.

L'autre écueil est de nature très différente, il tient à la révélation des motifs de non recours. Sous l'hypothèse que nous puissions constituer un échantillon d'étude, il est possible de distinguer la sous-population bénéficiaire de la prestation étudiée de celle n'y ayant pas recours. On peut alors espérer inférer de la comparaison de ces deux sous-populations les motifs de non recours (informationnel, économique). On peut associer la proximité d'un ménage à l'information pertinente au bénéfice présent ou passé d'autres prestations publiques, comme par exemple le bénéfice passé de la CMU-C. On peut aussi supposer que les ménages les moins sensibles au chèque santé sont ceux dont les revenus sont les plus faibles en raison de consommations incompressibles (c'est-à-dire faire l'hypothèse que l'élasticité de la demande au prix croît avec le revenu) ${ }^{7}$. Mais, d'une part, la révélation des motifs est nécessairement indirecte, d'autre part, sur des cibles étroites comme celle de l'ACS, la population éligible est très homogène, notamment du point de vue économique, ce qui laisse peu d'espoir d'obtenir des résultats.

Il est également possible de révéler directement les motifs de non recours en interrogeant les individus enquêtés ${ }^{8}$ mais il s'agit alors de réponses subjectives sensibles à la formulation des questions, utiles en elles-mêmes car permettant de dresser un panorama des motifs invoqués, mais qui ne renseignent qu'indirectement sur les effets attendus d'une campagne d'information ou d'une revalorisation du montant de l'aide.

Dans cette étude, nous adoptons une démarche directe d'évaluation des politiques publiques qui consiste à mesurer les effets sur le recours à l'ACS d'une modification du dispositif d'aide publique. La mise en œuvre d'une telle approche suppose bien sûr que l'on puisse observer de telles modifications. Comme on le constate à la lecture de l'encadré 1 , ceci a été le cas à plusieurs reprises depuis l'instauration de cette prestation en 2005, notamment concernant le montant du chèque. L'évaluation de ces aménagements aurait cependant demandé de s'appuyer sur l'observation d'une population témoin placée à l'écart des nouvelles règles. En effet, une comparaison avant/après du taux de recours à l'ACS est insuffisante du simple fait que le taux de recours à l'ACS poursuit une dynamique propre, indépendante des modifications législatives introduites, qu'il est impossible de prendre en compte de manière robuste sans observer une population témoin. Cette remarque est d'autant plus importante que l'ACS est un dispositif jeune, dont le taux de recours n'est pas stabilisé (cf. graphique, encadré 2).

En fait, l'évaluation rigoureuse de l'impact d'une mesure ou d'une politique publique sur le comportement d'un individu nécessiterait de pouvoir comparer le comportement d'un individu ayant bénéficié de cette mesure et le comportement qu'il aurait eu s'il n'en n'avait pas bénéficié. Ceci est bien sûr impossible sur la simple observation du comportement des individus une fois la mesure mise en place, puisque soit les individus en bénéficient, soit ils n'en bénéficient pas. Le modèle de résultats potentiels ou le cadre dit de Roy-Rubin (Roy, 1951; Rubin, 1974, cf. encadré 3) montre qu'il est toutefois possible d'inférer comment un individu se serait comporté s'il n'avait pas bénéficié

6. L'ACS dont le bénéfice suppose, à l'instar de la CMU-C, l'examen de l'ensemble des ressources du ménage (revenus du travail, du capital, prestations sociales, pensions reçues, y compris les avantages en nature procurés par le fait de disposer d'un logement valorisé de manière forfaitaire), sur les douze mois précédant la demande, n'échappe pas à cette difficulté.

7. Une méthode alternative consiste à identifier en population générale les élasticités-prix de la demande d'assurance et à en inférer l'impact attendu du chèque santé. Ceci suppose soit de pouvoir s'appuyer sur des données rassemblant des prix de contrats hétérogènes (voir les travaux sur données américaines : Auerbach et al., 2006, Thomas, 1995), soit d'introduire de fortes hypothèses de modélisation (comme Grignon et Kambia-Chopin, 2009, qui s'appuie sur une fonction d'utilité liant effet revenu et effet prix).

8. Voir Wittwer et al. (2010) pour une analyse du non recours à l'ACS sur données d'enquête. 
d'une mesure dans un cadre expérimental, solution que nous avons adoptée ici.

Le principe de cette expérimentation sociale est simple : identifier des ménages éligibles à l'ACS (ou plus précisément susceptibles de l'être) et constituer aléatoirement des groupes qui se verront proposer des chèques santé d'un montant différent ainsi que des accès différenciés à l'information. La constitution aléatoire des groupes permet alors de mesurer de manière robuste l'effet du montant du chèque et de l'accès à l'information. En effet, la parfaite homogénéité des groupes, en dehors du dispositif qu'il leur a

Encadré 3

\section{LE MODĖLE DE ROY-RUBIN}

Évaluer l'impact d'une mesure ou d'une politique publique sur le comportement d'un individu nécessite de pouvoir inférer comment l'individu se serait comporté s'il n'avait pas bénéficié de cette mesure. Le cadre d'analyse qui formalise ce problème est le modèle de résultats potentiels ou le cadre dit de RoyRubin (Roy, 1951 ; Rubin, 1974).

Ce modèle repose sur l'hypothèse qu'un individu ait accès à un traitement. Généralement le traitement correspond à une politique publique mise en place dont on souhaite évaluer l'impact. Pour cela, on définit une variable aléatoire $T$ représentant l'accès au traitement et prenant la valeur 1 lorsque l'individu $i$ bénéficie du traitement $\left(T_{i}=1\right)$ et la valeur 0 lorsqu'il n'en bénéficie pas $\left(T_{i}=0\right)$.

Afin de mesurer l'impact du traitement, sur la variable de résultat $Y$, on note $Y_{1 i}$ la valeur de $Y$ lorsque l'individu $i$ bénéficie du traitement et $Y_{0 i}$ lorsque ce même individu $i$ ne bénéficie pas du traitement.

L'effet propre (ou causal) du traitement pour l'individu $i$, noté $\Delta_{i}$, peut alors s'écrire comme la différence entre ses deux variables de résultats potentiels: $\Delta_{i}=Y_{1 i}-Y_{0 i}$. L'effet propre du traitement pour un individu donné n'est cependant pas observable par l'économètre: quand l'individu $i$ bénéficie du traitement, seul $Y_{1 i}$ est observé car $Y_{0 i}-i$.e. la valeur de $Y$ qui aurait été observée si l'individu n'avait pas été traité - n'est pas observable. $Y_{0 i}$ représente alors le résultat contrefactuel. De même, quand l'individu $i$ ne reçoit pas le traitement, $Y_{0 i}$ est observé et $Y_{1 i}$ ne l'est pas.

Le modèle de Roy-Rubin définit plusieurs paramètres permettant d'évaluer l'effet causal d'une mesure. Le paramètre le plus répandu dans la littérature est l'effet moyen du traitement sur la population qui en bénéficie (Average Treatment Effect on the Treated (ATT)). Plus précisément, on souhaite mesurer comment le traitement affecte en moyenne la variable de résultat par rapport à ce qu'elle serait si les personnes traitées n'avaient pas eu le traitement. Soit, formellement,

$\Delta_{A T T}=E\left[Y_{1}-Y_{0} \backslash T=1\right]=E\left[Y_{1} \backslash T=1\right]-E\left[Y_{0} \backslash T=1\right]$

Comme le contrefactuel $E\left[Y_{0} \backslash T=1\right]$ est inobservable, l'objectif est de lui trouver le meilleur substitut possible afin d'estimer sans biais l'ATT. Or, utiliser la moyenne de la variable de résultat sur les non traités $E\left[Y_{0} \backslash T=0\right]$ pour approximer le contrefactuel n'est habituellement pas une bonne solution car la variable d'affectation au traitement et la variable de résultat ne sont en général pas indépendantes si, par exemple, les individus qui ont un $\Delta_{i}$ positif participent plus souvent au traitement que les autres. Autrement dit, il existe un biais d'auto-sélection potentiel (ceux qui bénéficient du traitement sont ceux qui ont le plus à y gagner) égal à :

$$
\begin{aligned}
& E\left[Y_{1} \backslash T=1\right]-E\left[Y_{0} \backslash T=0\right]= \\
& \Delta_{A T T}+E\left[Y_{0} \backslash T=1\right]-E\left[Y_{0} \backslash T=0\right]
\end{aligned}
$$

Par conséquent, l'estimation de l'ATT est sans biais si et seulement si :

$E\left[Y_{0} \backslash T=1\right]-E\left[Y_{0} \backslash T=0\right]=0$ (ou encore si $\left(Y_{0} \perp T\right)$ )

On peut également s'intéresser à l'effet moyen du traitement sur l'ensemble de la population (Average treatment effect (ATE)) :

$$
\Delta_{A T E}=E\left[Y_{1}-Y_{0}\right]
$$

Dans ce cas, les deux contrefactuels doivent être approximés et la condition d'indépendance entre le traitement et le résultat porte alors sur les deux variables de résultats potentiels : $\left(Y_{1}, Y_{0} \perp T\right)$.

Dans le cadre d'une expérimentation sociale où le traitement est assigné de manière aléatoire, la variable de résultat et la variable de traitement sont indépendantes par construction, tant du point de vue des variables observables que des variables inobservables : on a donc

$E\left[Y_{0} \backslash T=1\right]=E\left[Y_{0} \backslash T=0\right]=E\left[Y_{0}\right]$ et

$E\left[Y_{1} \backslash T=1\right]=E\left[Y_{1} \backslash T=0\right]=E\left[Y_{1}\right]$.

Nous pouvons ainsi estimer l'effet moyen du traitement simplement en calculant la différence de moyenne de la variable de résultat entre le groupe traité et le groupe témoin :

$\Delta=E\left[Y_{1} \backslash T=1\right]-E\left[Y_{0} \backslash T=0\right]=\Delta_{A T E}=\Delta_{A T T}$.

De ce fait, si on observe une différence significative de la variable de résultat entre les groupes, cette différence est exclusivement attribuable au traitement.

On peut vérifier que les résultats bruts de l'expérimentation sont confirmés par l'estimation économétrique de l'effet de l'appartenance à chacun des groupes en contrôlant par les caractéristiques observées des assurés (cf. annexe). 
été proposé, garantit que toutes les différences de comportements observées peuvent être attribuées aux différences de dispositif.

Ce type d'expérimentation contrôlée nécessite la participation active des institutions concernées et de ce fait se révèle particulièrement coûteuse tant pour l'équipe en charge de l'évaluation que des institutions parties prenantes. Compte tenu des moyens mis en œuvre dans cette étude, l'expérimentation a été conduite au niveau local, celui de la Caisse primaire d'assurance maladie (CPAM) de Lille-Douai précisément.

\section{L'expérimentation}

C ette expérimentation sociale élaborée par l'université Paris-Dauphine a été mise en œuvre à la CPAM de Lille-Douai car celle-ci offrait, avant la mise en place de l'expérimentation, une prise en charge spécifique aux assurés sociaux se présentant à ses bureaux pour bénéficier de la CMU-C et relevant en fait de l'ACS. Au cours d'une réunion d'information à laquelle ils étaient invités, une majoration du montant du chèque santé financée sur le fonds de l'aide sociale leur était proposée. Ce dispositif d'aide spécifique visait donc à améliorer le recours à l'ACS mais ne concernait pas l'ensemble de la population éligible à l'ACS. Seules les personnes souhaitant bénéficier de la CMU-C, ayant essuyé un refus en raison de ressources trop élevées et ayant effectivement participé à la réunion d'information à laquelle elles étaient invitées pouvaient en bénéficier. En janvier 2009, la CPAM de Lille-Douai a accepté de mettre en œuvre une expérimentation sociale que nous avons élaborée afin de tester la généralisation à un échantillon de la population éligible d'une majoration de l'aide et de la conduite de réunions d'information, sur la base de ses pratiques antérieures.

Cette expérimentation s'est appuyée sur la campagne nationale d'information sur le dispositif ACS par courrier lancée en 2008, concrètement organisée au niveau de chaque CPAM. L'ensemble des assurés sociaux potentiellement éligibles à l'ACS et relevant de la CPAM de Lille-Douai ont été identifiés à la fin de l'année 2008 sur la base de leurs ressources de l'année 2006 ayant donné droit à des prestations par la Caisse d'allocations familiales de Lille (CAF) en 2008 à l'aide d'une requête informatique originellement conçue par l'Observatoire des non-recours aux droits et services de Grenoble
(Odenore) (Revil, 2008). Parmi l'ensemble des assurés sociaux de la CPAM de Lille-Douai potentiellement éligibles à l'ACS n'ayant pas fait valoir leur droit à la fin de l'année 2008, 4209 personnes ont été aléatoirement sélectionnées pour participer à cette expérimentation (cf. encadré 4).

Les individus du premier groupe (1 394 assurés), correspondant au groupe témoin, ont reçu un courrier (fin janvier 2009) les informant du dispositif national de l'ACS en vigueur à cette date; les individus du deuxième groupe (1 412 assurés), correspondant au premier groupe traité (groupe traité 1), ont reçu le même type de courrier (début février 2009) stipulant un montant majoré du chèque santé ; les individus du troisième groupe (1 403 assurés), correspondant au deuxième groupe traité (groupe traité 2), se sont vu proposer par courrier (en février/mars 2009) la même majoration du chèque santé et une invitation à une réunion d'information à la CPAM de Lille-Douai formalisée par l'envoi d'un second courrier la semaine suivante. La majoration de l'aide correspond à une augmentation de $62,5 \%$ à $75 \%$ du montant de l'aide nationale selon le groupe d'âge, les montants d'aide offerts à chaque groupe étant récapitulés dans le tableau 1.

Les courriers envoyés par la CPAM étaient accompagnés des formulaires que les éligibles potentiels étaient invités à retourner pour évaluation de leur éligibilité effective à l'ACS. En effet, les fichiers transmis par la CAF ne permettaient de cibler que la population susceptible d'être éligible sans garantir que les ménages le soient effectivement, puisque, comme précisé plus haut, les fichiers de la CAF ont été constitués sur la base des revenus de l'année 2006 et des allocations perçues en 2008 alors que l'éligibilité à l'ACS est déterminée sur les ressources des douze derniers mois.

Les réunions d'information se sont tenues en amont, avant instruction par la CPAM des dossiers. Une douzaine de réunions ont été organisées de février à avril 2009, au rythme d'environ deux réunions par semaine le jeudi matin et le samedi matin. C'est la raison pour laquelle les courriers du deuxième groupe traité ont été envoyés par vagues successives sur deux mois afin de pouvoir gérer le flux des ménages répondant favorablement à l'invitation à la réunion d'information.

Ces réunions d'information visaient à informer les assurés sociaux sur le dispositif de l'ACS 
et les formalités nécessaires pour en bénéficier. Elles étaient conduites par une assistante sociale rémunérée spécifiquement pour cette tache et ont été observées par une anthropologue ${ }^{9}$. Par ailleurs, les services de la CPAM ont été sollicités pour organiser l'envoi des courriers propres à l'expérimentation et collecter les informations concernant le retour des dossiers et les notifications $\mathrm{ACS}^{10}$.

Le retour des dossiers à la CPAM et les notifications effectives d'ACS ont été observés entre le
21 janvier (date de l'envoi de la première vague des courriers) et le 30 juillet 2009 (date de fin de l'expérimentation) par le service des prestations de la CPAM de Lille-Douai. Les données recueillies par la CPAM permettent de connaître

9. On trouvera dans Wittwer et al. (2010) une description de l'analyse anthropologique qualitative des réunions. L'encadré 6 rend compte des principales conclusions.

10. On peut se référer à Wittwer et al. (2010) pour plus d'informations sur le personnel mobilisé à la CPAM de Lille-Douai pour la mise en œuvre pratique de l'expérimentation et une analyse qualitative de leur opinion sur l'expérimentation.

\section{Encadré 4}

\section{IDENTIFICATION DES ASSURÉS ET DÉFINITION DE LA POPULATION EXPÉRIMENTÉE}

La population expérimentée se compose d'assurés de la CPAM de Lille-Douai identifiés comme potentiellement éligibles à l'ACS à partir de leurs ressources 2006. La requête informatique sur les fichiers de la CAF (Caisse d'allocations familiales) élaborée dans le cadre de la campagne nationale d'information sur le dispositif ACS lancée en 2008 a été utilisée. Cette requête a été lancée sur les fichiers de la CAF dans la métropole lilloise en décembre 2008. Sur la base de la déclaration de leurs ressources de l'année 2006 ayant donné droit à des prestations délivrées en 2008 par la CAF de Lille, elle a permis d'identifier 7436 personnes potentiellement éligibles à l'ACS et n'ayant pas fait valoir leur droit. Cette population éligible a été réduite (pour raisons financières) par échantillonnage aléatoire pour définir une population d'expérimentation comprenant 5000 assurés répartis entre trois groupes.

La même requête informatique a été conduite dans l'ensemble des CPAM dans le but d'informer de leur droit l'ensemble de la population potentiellement éligible à l'ACS. Une requête affinée est aujourd'hui effectuée chaque mois par les CAF qui transmettent aux CPAM un fichier de nouveaux éligibles potentiels. Il est important de souligner qu'en utilisant les fichiers de la CAF pour identifier les éligibles potentiels, nous restreignons l'analyse aux assurés sociaux bénéficiant d'une prestation sociale de la CAF. Sans pouvoir avancer de chiffre précis, il est vraisemblable que l'échantillon ainsi sélectionné soit largement représentatif de la population éligible. Néanmoins, nous sélectionnons une population ayant déjà recours à l'aide publique, ce qui écarte de fait la population n'ayant « systématiquement » pas recours aux prestations publiques ainsi que la population éligible à l'ACS et non éligible aux prestations de la CAF (les personnes âgées propriétaires de leur logement par exemple).

Il faut garder à l'esprit que l'éligibilité à l'ACS s'évalue au regard des ressources du ménage et que la prestation elle-même est une prestation attribuée au ménage. Par ailleurs, certains ménages (les couples bi-actifs en particulier) sont composés de plusieurs assurés sociaux identifiés par la CAF comme potentiellement éligibles. Comme les courriers nationaux pour informer les assurés sur le dispositif ACS, sont envoyés par la CPAM à chaque assuré, certains ménages ont reçu plusieurs courriers. Dans notre cadre expérimental, cette éventualité est problématique. En effet, deux assurés sociaux affectés aléatoirement dans des groupes différents mais appartenant au même ménage, peuvent avoir reçu deux propositions d'aide différentes. Afin de corriger ce biais de contamination, nous avons retiré de l'analyse expérimentale tous les assurés sociaux appartenant à un même ménage et ayant reçu des courriers différents. Par ailleurs, nous avons retenu aléatoirement un assuré au sein des ménages dans lesquelles plusieurs assurés sociaux ont reçu le même courrier. En conséquence, les assurés sociaux appartenant à un ménage bi-actif sont globalement sous représentés mais identiquement sous-représentés dans chaque groupe. L'échantillon des individus est ainsi réduit à 4209 assurés sociaux.

Tableau 1

Montants d'ACS offerts dans le cadre de l'expérimentation

\begin{tabular}{|l|c|c|c|}
\hline \multirow{2}{*}{} & \multicolumn{3}{|c|}{ Montants offerts par personne } \\
\cline { 2 - 4 } & Moins de 25 ans & Entre 25 et 59 ans & 60 ans et plus \\
\hline Groupe & $100 €$ & $200 €$ & $400 €$ \\
Témoin & $175 €$ & $350 €$ & $650 €$ \\
Traité 1 \& Traité 2 & \multicolumn{3}{|c|}{} \\
\hline
\end{tabular}

Lecture: les ménages affectés au groupe témoin ont reçu une proposition d'ACS d'un montant de $100 €$ pour chaque personne de moins de 25 ans, $200 €$ pour chaque personne âgée entre 25 et 59 ans et $400 €$ pour chaque membre du ménage de 60 et plus. Champ : ensemble de la population dans le champ d'expérimentation (échantillonnage aléatoire sur les fichiers de la CAF dans la métropole lilloise).

Source : données des auteurs. 
pour chaque assuré appartenant à l'échantillon expérimenté : son groupe d'appartenance; si un dossier de demande ACS a été complété et adressé ou non à la CPAM; si après examen du dossier, l'ACS a été notifiée ou non par les services de la CPAM, c'est-à-dire si l'ACS a été accordée ; en cas de refus, si celui-ci est motivé par des ressources trop élevées ou au contraire trop faibles, ce qui a conduit à ouvrir les droits à la CMU-C. Enfin, pour les assurés appartenant au groupe traité 2 , l'information sur la venue à la réunion a été conservée.

Ces données ont ensuite été appariées aux données administratives de la CPAM, contenant des informations sur l'âge, le sexe, le régime de remboursement au 31 décembre 2008 (actif, retraité, bénéficiaire d'une pension d'invalidité, d'une rente ou de l'allocation adulte handicapé (AAH), bénéficiaire d'une exonération du ticket modérateur dans le cadre d'une ALD), les dépenses de soins ambulatoires en 2008, ainsi que sur le statut vis-à-vis de la complémentaire santé avant le début de l'expérimentation et le fait d'avoir bénéficié de la CMU-C en 2007 (cf. encadré 5).

La lecture du tableau 2 offre une première description des données rassemblées. On vérifie, en premier lieu, que la constitution aléatoire des groupes expérimentés conduit à des distributions des variables observées très similaires ${ }^{11}$. Les assurés sociaux constituant l'échantillon sont, à part égale, des hommes et des femmes, $15 \%$ ont au moins un ayant-droit de moins de 3 ans ${ }^{12}$. Une grande proportion d'entre eux, près de $80 \%$, sont âgés de 25 à 59 ans alors que les moins de 25 ans représentent moins de $10 \%$ de chaque groupe. Si l'on considère le régime de l'assuré, $60 \%$ sont actifs, près de $25 \%$ sont bénéficiaires d'une pension d'invalidité et $15 \%$ disposent d'une pension de retraite. On note

11. Les tests de Chi deux effectués ne permettent pas de rejeter l'hypothèse nulle d'identité des distributions.

12. Il est important de noter que nous observons ici la "grappe " d'ayants droits et non la composition du ménage.

\section{Encadré 5}

\section{LES DONNÉES ADMINISTRATIVES DE LA CPAM}

Pour chaque assuré dans le champ de l'expérimentation, nous disposons d'informations enregistrées par la CPAM de Lille-Douai avant le début de l'expérimentation (fin 2008). II s'agit de caractéristiques socio-démographiques, mais aussi du régime de l'assuré à la CPAM, son profil par rapport aux soins et aux remboursements, et s'il bénéficie d'une couverture complémentaire.

\section{Caractéristiques socio-démographiques}

Le sexe de l'assuré et son âge au $1^{\text {er }}$ janvier 2009. Si l'assuré a un ou des ayant-droit(s) de moins de 3 ans en 2008.

\section{Le régime CPAM}

La CPAM définit différents régimes en fonction de la situation professionnelle ou personnelle de l'assuré. Nous les avons regroupés comme suit au 31 décembre 2008 :

- Actif : il s'agit des salariés du privé et des établissements publics ainsi que les artistes du spectacle, les artistes auteurs et les praticiens et auxiliaires médicaux, en emploi ou au chômage.

- Invalide : concerne (1) les bénéficiaires d'une pension d'invalidité (incapacité de reprendre son travail après un accident ou une maladie invalidante d'origine non professionnelle), (2) les bénéficiaires d'une rente dans le cadre d'une maladie professionnelle ou à la suite d'un accident du travail, (3) les bénéficiaires d'une allocation aux adultes handicapés (AAH) (cf. CAF)
- Retraité : il s'agit des bénéficiaires d'une pension de retraite ou d'une pension de réversion.

\section{Les dépenses de soins en 2008}

Nous disposons pour chaque assuré des dépenses de soins totales en ambulatoire remboursées par la CPAM en 2008. Le système d'information ne permet pas encore aujourd'hui de collecter des informations précises sur les consommations individuelles à l'hôpital.

\section{La prise en charge à $100 \%$ dans le cadre d'une Affection de longue durée (ALD)}

De même les données de remboursements de la CPAM nous renseignent sur le bénéfice d'une prise en charge à $100 \%$ dans le cadre d'une ALD pour l'année 2008.

\section{La couverture complémentaire}

La CPAM a non seulement des informations sur les bénéficiaires de la CMU-C, et nous avons retenu l'information relative au bénéfice de la CMU-C au cours de l'année 2007, mais sait également si l'assuré est couvert par une complémentaire santé (CS). La norme informatique d'échange d'informations NOEMIE (Norme ouverte d'échange entre la maladie et les intervenants extérieurs) entre l'Assurance maladie et les organismes de CS permet de collecter de telles informations. II faut cependant noter que tous les organismes de CS ne sont pas affiliés à NOEMIE, ce qui conduit à une sous estimation du taux de recours à une CS. 
enfin que le mois précédant l'expérimentation, en décembre 2008, un assuré sur trois n'est pas couvert par une complémentaire santé ${ }^{13}$ et $50 \%$ de la population expérimentée ont engagé des dépenses supérieures à 700 euros en 2008.

\section{Évaluation des traitements}

L' ensemble de ces données rassemblées permet d'évaluer les effets de l'envoi des courriers spécifiques aux individus des groupes traités sur le recours à l'ACS. Par commodité de langage, nous parlerons par la suite des effets des «traitements » (soit de la majoration du chèque santé, traitement 1 , soit de la majoration du chèque santé avec invitation à une réunion, traitement 2 ) et de la « réaction aux traitements » des assurés sociaux.
Pour évaluer l'efficacité des traitements, nous centrons notre attention sur deux variables d'intérêt: le taux de retour de dossiers par rapport au nombre de dossiers envoyés et le taux d'ACS notifiées, c'est-à-dire la proportion de chèque santé délivrés par la CPAM de Lille-Douai par rapport au nombre de dossiers envoyés. La constitution aléatoire des groupes traités et du groupe témoin autorise des comparaisons directes des variables d'intérêt afin d'évaluer les effets des traitements (cf. encadré 6). Ainsi, la comparaison du nombre de dossiers retournés à la CPAM et du nombre d'ACS effectivement accordées entre

13. Ce taux est bien supérieur aux évaluations sur données d'enquête pour ce type de population (Ce taux est de $19 \%$ si on fait une approximation de la population par le premier décile de revenu et de $14 \%$ pour le deuxième décile (Arnould et Vidal, 2008)). La montée en charge progressive de la norme d'échange Noémie explique certainement pour une partie cette différence: tous les organismes de complémentaire santé ne participant pas en décembre 2008 à ce système de collecte d'information.

Tableau 2

Caractéristiques des assurés avant l'expérimentation

\begin{tabular}{|c|c|c|c|c|c|c|c|c|}
\hline & \multicolumn{8}{|c|}{ Groupes } \\
\hline & \multicolumn{2}{|c|}{ Témoin } & \multicolumn{2}{|c|}{ Traité 1} & \multicolumn{2}{|c|}{ Traité 2} & \multicolumn{2}{|c|}{ Total } \\
\hline & Effectifs & $\%$ & Effectifs & $\%$ & Effectifs & $\%$ & Effectifs & $\%$ \\
\hline \multicolumn{9}{|l|}{ Age de l'assuré } \\
\hline Moins de 25 ans & 105 & 7,5 & 113 & 8,0 & 108 & 7,7 & 326 & 7,7 \\
\hline 25-59 ans & 1048 & 75,2 & 1056 & 74,8 & 1040 & 74,1 & 3144 & 74,7 \\
\hline 60 ans et plus & 241 & 17,3 & 243 & 17,2 & 255 & 18,2 & 739 & 17,6 \\
\hline \multicolumn{9}{|l|}{ Sexe de l'assuré } \\
\hline Homme & 679 & 48,7 & 691 & 48,9 & 693 & 49,4 & 2063 & 49,0 \\
\hline Femme & 715 & 51,3 & 721 & 51,1 & 710 & 50,6 & 2146 & 51,0 \\
\hline \multicolumn{9}{|c|}{ Ayant-droit de moins de 3 ans } \\
\hline Oui & 206 & 14,8 & 211 & 14,9 & 217 & 15,5 & 634 & 15,1 \\
\hline Non & 1188 & 85,2 & 1201 & 85,1 & 1186 & 84,5 & 3575 & 84,9 \\
\hline \multicolumn{9}{|l|}{ Régime de l'assuré } \\
\hline Actif & 845 & 60,6 & 857 & 60,7 & 865 & 61,6 & 2567 & 61,0 \\
\hline Invalide & 339 & 24,3 & 349 & 24,7 & 338 & 24,1 & 1026 & 24,4 \\
\hline Retraité & 210 & 15,1 & 206 & 14,6 & 200 & 14,3 & 616 & 14,6 \\
\hline \multicolumn{9}{|c|}{ Dépenses en soins ambulatoires en 2008} \\
\hline $0 €$ à $199 €$ & 374 & 26,8 & 350 & 24,8 & 362 & 25,7 & 1086 & 25,8 \\
\hline $200 €$ à $699 €$ & 340 & 24,4 & 365 & 25,9 & 355 & 25,3 & 1060 & 25,2 \\
\hline $700 €$ à $1999 €$ & 338 & 24,3 & 333 & 23,6 & 357 & 25,5 & 1028 & 24,4 \\
\hline$>=2000 €$ & 342 & 24,5 & 364 & 25,7 & 329 & 23,5 & 1035 & 24,6 \\
\hline \multicolumn{9}{|c|}{ Soins en ALD en 2008} \\
\hline Oui & 385 & 27,6 & 398 & 28,2 & 407 & 29,0 & 1190 & 28,3 \\
\hline Non & 1009 & 72,4 & 1014 & 71,8 & 996 & 71,0 & 3019 & 71,7 \\
\hline \multicolumn{9}{|c|}{ Complémentaire santé en décembre 2008} \\
\hline Oui & 927 & 66,5 & 935 & 66.2 & 923 & 65,8 & 2785 & 66,2 \\
\hline Non & 467 & 33,5 & 477 & 33,8 & 480 & 34,2 & 1424 & 33,8 \\
\hline \multicolumn{9}{|c|}{ CMUC en décembre 2007} \\
\hline Oui & 98 & 7,0 & 100 & 7,1 & 91 & 6,5 & 289 & 6,9 \\
\hline Non & 1296 & 93,0 & 1312 & 92,9 & 1312 & 93,5 & 3920 & 93,1 \\
\hline Total & 1394 & 100,0 & 1412 & 100,0 & 1403 & 100,0 & 4209 & 100,0 \\
\hline
\end{tabular}

Lecture : dans le groupe témoin, 105 (7,5\%) assurés sont âgés de moins de 25 ans au $1^{\text {er }}$ janvier 2009

Champ : ensemble de la population dans le champ d'expérimentation (échantillonnage aléatoire sur les fichiers de la CAF dans la métropole lilloise).

Source : données et calculs des auteurs. 
le groupe témoin et le groupe traité 1 permet de tester l'effet de la majoration du chèque santé ; d'autre part, la comparaison de ces indicateurs entre le groupe traité 1 et traité 2 permet de tester l'effet de l'invitation à la réunion d'information.

\section{Effet faible mais significatif de la majoration du chèque santé sur le recours à l'ACS}

La réaction des assurés expérimentés à l'envoi des courriers par la CPAM de Lille-Douai est tout d'abord mesurée par le nombre de dossiers complets retournés à la CPAM. Sur les 4209 assurés, 701 dossiers complets ont été réceptionnés et ont fait l'objet d'une suite, soit un taux de retour de $17 \%$ (cf. tableau 3). Le taux de retour est globalement assez modeste. Pour le groupe témoin, $16 \%$ des assurés ont retourné un dossier complet pour obtenir l'ACS (soit 222 dossiers). Les assurés s'étant vu proposer uniquement l'aide majorée (groupe traité 1) ont significativement plus souvent complété un dossier que les assurés du groupe témoin (au seuil de significativité de $5 \%$ ) avec $18,6 \%$ de taux de retours (cf. tableau 3). La majoration du chèque santé semble ainsi avoir un impact sur la probabilité de déposer un dossier, bien que cet impact soit limité.

Cet impact peut être mesuré par l'élasticité de la probabilité de déposer un dossier complet par rapport au montant du chèque. Cette élasticité est calculée comme le rapport du taux de croissance de la probabilité de retourner un dossier complet entre le groupe témoin et le groupe 1 d'une part, et du taux de croissance du montant $\mathrm{du}$ chèque santé entre le montant national et le montant majoré pour les moins de 60 ans $^{14}$. Elle s'établit à 0,22 (tableau 6). Cet indica-

14. Nous adoptons un taux de croissance du chèque de $75 \%$ alors que ce taux s'élève à $62,5 \%$ pour les plus de 60 ans. Nous faisons ainsi le choix du calcul d'une élasticité globale minorée plutôt que le calcul d'élasticité par tranche d'âge car le faible effectif des plus de 60 ans ne permet pas de calculer une élasticité avec suffisamment de précision.

Encadré 6

\section{L'ACCÈS À L'ACS DU POINT DE VUE DES USAGERS}

L'analyse qualitative a permis de recueillir les besoins et les attentes des personnes ciblées par l'ACS par deux types de méthodes complémentaires: d'une part l'observation de réunions d'information et, d'autre part, la conduite d' entretiens approfondis avec des bénéficiaires potentiels dont certains étaient venus à la réunion (voir Wittwer et al. 2010, pour une présentation détaillée).

Deux profils de personnes participant aux réunions ont pu être identifiés :

- des individus qui s'approprient les éléments d'information qui leur sont fournis et évaluent par euxmêmes s'ils sont éligibles :

o certains d'entre eux ont pu constater lors de la réunion que ce n'était pas le cas et dès lors n'ont pas déposé de dossier ;

- des individus qui avaient des difficultés à comprendre le dispositif, les barèmes, les démarches à entreprendre :

o la réunion n'a pas suffi à lever leurs difficultés: certains ont certainement besoin d'un accompagnement individualisé (lors de la réunion, l'animatrice ne pouvait pas assurer cette aide) ;

o cela ne préjuge pas du non dépôt du dossier car quelques uns ont été chercher cet accompagnement auprès d'une assistante sociale ou d'un agent d'accueil de la Sécurité sociale, comme les entretiens le démontrent;

o d'autres ont abandonné les démarches.

Ces éléments révèlent qu'au-delà de l'information sur l'existence du dispositif, la complexité du dispositif et des démarches à entreprendre pour y accéder découragent une partie des personnes éligibles. L'ACS est un dispositif complexe. Pour en comprendre l'intérêt, un prérequis est nécessaire, comprendre le système de protection sociale et les modalités de remboursement des soins (par l'Assurance maladie obligatoire et par les organismes complémentaires).

De plus, elle suppose deux niveaux de démarche. Le dépôt du dossier de demande (équivalent au dépôt de dossier $\mathrm{CMU}-\mathrm{C}$ ) et dans une deuxième phase, le choix d'une complémentaire santé. Cette dernière suppose de se repérer dans une offre de marché abondante et concurrente, avec des contrats à plusieurs niveaux, difficiles à interpréter, notamment pour une partie de cette population en difficulté avec le jargon administratif et a fortiori assurantiel. Ce choix doit en outre être mis en regard des besoins de santé dans le futur, difficiles à évaluer.

L'observation des réunions permet également de mettre en avant un des obstacles au dispositif à savoir les difficultés à comprendre le langage administratif. Ainsi, nombre de personnes sont venues sans trop savoir quel était l'objet de la réunion. Une partie d'entre elles pensait être convoquée, d'autres cherchaient à glaner des informations sur une aide possible et souhaitaient "ne pas passer à côté d'un droit ", sans saisir correctement la teneur de cette aide. La communication par courrier entre la caisse et les usagers reste donc problématique pour cette frange de la population et constitue un obstacle à la diffusion large de l'information. 
teur rend compte de la sensibilité des assurés sociaux au montant du chèque santé, et montre ici qu'une augmentation du chèque santé de $10 \%$ augmente la probabilité de compléter un dossier de $2,2 \%$.

\section{Seule la moitié des individus ayant complété un dossier se voit délivrer un chèque santé}

Au-delà des dossiers complétés, on peut s'interroger sur la proportion des individus ayant reçu un chèque santé dans le cadre de l'expérimentation. En effet, l'ACS n'a pas été accordée par la CPAM à tous les assurés ayant renvoyé un dossier, en raison de ressources en dehors des seuils d'éligibilité. $\mathrm{Au}$ total, $55 \%$ des dossiers retournés complets ont donné droit à l'ACS (cf. tableau 4), $10 \%$ ont donné droit à la CMU-C car les ressources des ménages étaient inférieures au seuil d'éligibilité de l'ACS et $36 \%$ ont été refusés pour cause de ressources trop élevées (cf. tableau 5). Parmi les 4209 assurés inclus dans l'expérimentation, $9 \%$ ont donc effectivement obtenu l'ACS, $2 \%$ la CMU-C, $6 \%$ se sont vu refuser l'ACS et la CMU-C et $83 \%$ n'ont fait aucune demande.

La comparaison du nombre d'ACS effectivement accordées selon les groupes conduit à des conclusions assez comparables à celles tirées de l'analyse du nombre de dossiers retournés. Néanmoins, l'écart entre le groupe témoin et le groupe traité 1 est sensiblement accentué. Le taux d'accords ACS par rapport au nombre d'assurés expérimentés est ainsi de $11 \%$ dans le groupe traité 1 contre $8 \%$ dans le groupe témoin. L'élasticité de la probabilité, définie comme le rapport du taux de croissance de la probabilité d'obtenir l'ACS et du taux de croissance du montant du chèque entre les groupes témoin et traité 1 , s'établit à 0,49

Tableau 3

Nombre et taux de dossiers retournés par groupe

\begin{tabular}{|c|c|c|c|c|c|}
\hline \multirow{2}{*}{ Groupes } & \multicolumn{2}{|c|}{ Dossiers complets } & \multirow[t]{2}{*}{ IC $95 \%$} & \multicolumn{2}{|c|}{ Nombre d'assurés } \\
\hline & Effectifs & $\%$ & & Effectifs & $\%$ \\
\hline Témoin & 222 & 15,9 & $(14,0 ; 17,8)$ & 1394 & 100,0 \\
\hline Traité 1 & 262 & 18,6 & $(16,5 ; 20,6)$ & 1412 & 100,0 \\
\hline Traité 2 & 217 & 15,5 & $(13,6 ; 17,4)$ & 1403 & 100,0 \\
\hline dont Avec réunion & 35 & 28,0 & $(20,0 ; 36,0)$ & 125 & 100,0 \\
\hline Sans réunion & 182 & 14,2 & $(12,3 ; 16,2)$ & 1278 & 100,0 \\
\hline Total & 701 & 16,7 & $(15,5 ; 17,8)$ & 4209 & 100,0 \\
\hline
\end{tabular}

Lecture : dans le groupe témoin, parmi les 1394 assurés, 222 (15,9\%) ont retourné un dossier complet de demande d'ACS. La proportion de dossiers complétés dans le groupe témoin (15,9\%) appartient à l'intervalle (14\%;17,8 \%) avec un niveau de confiance de $95 \%$. Au sein des 1403 individus affectés au groupe traité 2 à qui une réunion d'information a été proposée, 125 s'y sont rendus. Parmi ces 125 individus, 35 (28 \%) ont retourné un dossier complet de demande d'ACS.

Champ : ensemble de la population dans le champ d'expérimentation (échantillonnage aléatoire sur les fichiers de la CAF dans la métropole lilloise)

Source : données et calculs des auteurs.

Tableau 4

ACS accordées par groupe

\begin{tabular}{|c|c|c|c|c|c|}
\hline Groupes & Effectifs & $\begin{array}{l}\text { Proportion du nombre total d'assurés } \\
\text { dans le champ d'expérimentation } \\
\text { en } \%\end{array}$ & IC $95 \%$ & $\begin{array}{l}\text { Proportion du nombre } \\
\text { de dossiers complets } \\
\text { en } \%\end{array}$ & IC $95 \%$ \\
\hline Témoin & 110 & 7,9 & $(6,5 ; 9,3)$ & 49,6 & $(42,9 ; 56,2)$ \\
\hline Traité 1 & 152 & 10,8 & $(9,1 ; 12,4)$ & 58,0 & $(52,0 ; 64,0)$ \\
\hline Traité 2 & 125 & 8,9 & $(7,4 ; 10,4)$ & 57,6 & $(51,0 ; 64,2)$ \\
\hline dont Avec réunion & 22 & 17,6 & $(10,8 ; 24,4)$ & 62,9 & $(46,0 ; 79,6)$ \\
\hline Sans réunion & 103 & 8,1 & $(6,6 ; 9,6)$ & 56,6 & $(49,3 ; 63,9)$ \\
\hline Total & 387 & 9,2 & $(8,3 ; 10,1)$ & 55,2 & $(51,5 ; 58,9)$ \\
\hline
\end{tabular}

Lecture : parmi les 1394 assurés du groupe témoin, 110 ont eu droit à l'ACS, soit 7,9\% des courriers envoyés et 49,6 \% des dossiers complétés.

Champ : ensemble de la population dans le champ d'expérimentation d'expérimentation (échantillonnage aléatoire sur les fichiers de la CAF dans la métropole lilloise).

Source : données et calculs des auteurs. 
(cf. tableau 6), soit un niveau sensiblement plus élevé que celle calculée sur la base des taux de retour des dossiers complets.

Cette augmentation de l'élasticité est la conséquence du fait que le taux d'acceptation des dossiers complets est plus faible dans le groupe témoin que dans les groupes traités. En effet, le taux d'accords ACS par rapport au nombre de dossiers complets envoyés est de $58 \%$ dans le groupe traité 1 contre $49 \%$ seulement dans le groupe témoin (cf. tableau 4). L'aide exceptionnelle offerte aux individus des groupes traités semble avoir sélectionné plus précisément les assurés effectivement éligibles, c'est-à-dire les plus pauvres parmi les assurés expérimentés, puisque le taux de refus en raison de ressources trop élevées est plus faible dans les groupes traités 1 et 2 que dans le groupe témoin (cf. tableau 5).

\section{L'invitation à la réunion d'information annule l'effet de la majoration du chèque santé}

Pour le groupe traité 2, dont les membres ont reçu une invitation à une réunion d'information ainsi qu'une proposition d'ACS majorée, le taux de retour de dossiers complets s'élève à $15,5 \%$. Ce taux est légèrement inférieur à celui du groupe témoin, mais pas de manière significative. En revanche, ce taux est significativement moins élevé que celui du groupe traité 1 (au seuil significativité de 5\%). De manière assez inattendue, l'invitation à la réunion a donc constitué un frein au recours à

Tableau 5

Les motifs de refus d'accord ACS

\begin{tabular}{|c|c|c|c|c|c|c|}
\hline \multirow[b]{2}{*}{ Groupes } & \multicolumn{3}{|c|}{ CMU-C (ressources < plafond) } & \multicolumn{3}{|c|}{ Ressources $>$ plafond } \\
\hline & Effectifs & $\begin{array}{c}\text { Proportion } \\
\text { du nombre total } \\
\text { d'assurés dans } \\
\text { le champ } \\
\text { d'expérimentation } \\
\text { en \% }\end{array}$ & $\begin{array}{c}\text { Proportion } \\
\text { du nombre de } \\
\text { dossiers complets } \\
\text { en } \%\end{array}$ & Effectifs & $\begin{array}{c}\text { Proportion } \\
\text { du nombre total } \\
\text { d'assurés dans } \\
\text { le champ } \\
\text { d'expérimentation } \\
\text { en \% }\end{array}$ & $\begin{array}{c}\text { Proportion } \\
\text { du nombre de } \\
\text { dossiers complets } \\
\text { en } \%\end{array}$ \\
\hline Témoin & 25 & 1,8 & 11,3 & 87 & 6,2 & 39,2 \\
\hline Traité 1 & 25 & 1,8 & 9,5 & 85 & 6,0 & 32,4 \\
\hline Traité 2 & 21 & 1,5 & 9,7 & 71 & 5,1 & 32,7 \\
\hline dont Avec réunion & 2 & 1,6 & 5,7 & 11 & 8,8 & 31,4 \\
\hline Sans réunion & 19 & 1,5 & 10,4 & 60 & 4,7 & 33,0 \\
\hline Total & 71 & 1.7 & 10,1 & 243 & 5,8 & 34,7 \\
\hline
\end{tabular}

Lecture : pour le groupe témoin, 25 assurés ont obtenu un accord CMU-C, soit 1,8 \% des assurés du champ de l'expérience et 11,3\% des dossiers complétés.

Champ : ensemble de la population dans le champ d'expérimentation d'expérimentation (échantillonnage aléatoire sur les fichiers de la CAF dans la métropole lilloise).

Source : données et calculs des auteurs.

Tableau 6

Elasticités de la demande d'ACS au montant de l'aide et selon le statut vis-à-vis de la complémentaire santé en décembre 2008

\begin{tabular}{|c|c|c|c|c|c|c|c|c|}
\hline & \multicolumn{4}{|c|}{ Dossier complété } & \multicolumn{4}{|c|}{ ACS notifiée } \\
\hline & \multicolumn{2}{|c|}{ Taux de retour ( \%) } & \multicolumn{2}{|c|}{ Élasticité (E) } & \multicolumn{2}{|c|}{ Taux de retour ( \%) } & \multicolumn{2}{|c|}{ Élasticité (E) } \\
\hline & Témoin & Traité 1 & $E$ & IC $95 \%$ & Témoin & Traité 1 & $\mathrm{E}$ & IC $95 \%$ \\
\hline Ensemble & 15,9 & 18,6 & 0,22 & $(-0,01 ; 0,49)$ & 7,9 & 10,8 & 0,49 & $(0,11 ; 0,96)$ \\
\hline \multicolumn{9}{|c|}{ Selon le statut par rapport à la CS en 2008} \\
\hline Couvert par une CS & 16,4 & 19,0 & 0,21 & $(-0,06 ; 0,55)$ & 8,2 & 11,1 & 0,48 & $(-0,03 ; 1,06)$ \\
\hline Non couvert par une CS & 15,0 & 17,6 & 0,23 & $(-0,16 ; 0,76)$ & 7,3 & 10,1 & 0,51 & $(-0,12 ; 1,47)$ \\
\hline
\end{tabular}

Lecture : élasticités calculées en faisant le rapport entre, le taux de croissance de la probabilité de constituer une dossier complet (d'obtenir l'ACS) entre le groupe témoin et le groupe traité 1, d'une part, et, le taux de croissance du chèque santé entre le montant national et le montant majoré pour les moins de 60 ans (ce taux étant légèrement inférieur pour les plus de 60 ans) d'autre part. Pour les assurés sans complémentaire santé en 2008, une augmentation du prix de $10 \%$ augmente la probabilité de retourner un dossier de 2,3\%. Champ : ensemble de la population dans le champ d'expérimentation d'expérimentation (échantillonnage aléatoire sur les fichiers de la CAF dans la métropole lilloise).

Source : données et calculs des auteurs. 
l'ACS, qui a annulé l'effet positif de la majoration du chèque santé.

Il convient toutefois d'éclairer ce résultat au regard de la participation effective à la réunion. Parmi les 1403 assurés du groupe traité 2, seuls 125 se sont effectivement rendus à la réunion d'information à laquelle ils étaient invités (soit $9 \%$ ). Parmi ces derniers, 35 ont retourné un dossier complet. Le taux de retour de dossiers complets s'établit donc à $28 \%$ parmi les personnes ayant effectivement participé à la réunion. Au contraire, parmi les 1278 assurés du groupe traité 2 qui ne sont pas allés à la réunion d'information, le taux de retour n'est que de $14,2 \%$.

On peut avancer l'idée qu'une partie des assurés du groupe traité 2 ne s'étant pas rendus à la réunion, ont tenu pour obligatoire leur présence et n'ont donc pas jugé bon d'entreprendre des démarches dès lors qu'ils ne pouvaient s'y rendre. Le taux de retour important parmi les personnes ayant participé à la réunion est plus difficile à interpréter. Si la tentation est forte de penser que les réunions d'information ont favorisé le recours à l'ACS des personnes qui y ont effectivement participé, il est fort probable que ce recours plus important à l'ACS s'explique simplement par l'intérêt plus marqué pour le dispositif de l'ACS des personnes qui ont effectivement assisté à la réunion ${ }^{15}$. Ainsi l'analyse des déterminants de la participation à la réunion montre que les individus présents ont un profil particulier : ils sont plus âgés, plus souvent pris en charge à $100 \%$ par la Sécurité sociale dans le cadre d'une Affection de longue durée, sans complémentaire santé en début d'expérimentation et ont engagé des dépenses de santé plus importantes en 2008 (cf. Wittwer et al., 2010).

\section{Un dispositif qui a du mal à toucher sa cible en raison notamment de sa complexité}

Cette expérimentation montre enfin la difficulté de mettre en place une campagne d'information sur ce dispositif, qui au final touche difficilement sa cible. En dépit de l'envoi d'un courrier à l'ensemble des assurés sociaux potentiellement éligibles, moins d'une personne sur cinq a finalement retourné un dossier pour demander l'ACS (taux de retour de $17 \%$ ). Autre fait troublant, le taux de retour des dossiers ainsi que sa sensibilité au montant de l'aide sont très similaires parmi les personnes initialement couvertes par une complémentaire santé $(66 \%$ de l'échantillon) et les personnes initialement non couvertes (33\%). En effet, le taux de retour de dossier des personnes initialement couvertes est de $16,4 \%$ dans le groupe témoin et $19 \%$ dans le groupe traité 1 contre respectivement $15 \%$ et $17,6 \%$ pour les personnes non couvertes, ces différences n'étant pas significatives (cf. tableau 6 ). De même on ne note aucune différence significative de l'élasticité du taux de recours au montant du chèque selon la couverture $(0,23$ pour les personnes initialement couvertes contre 0,21 pour les non couvertes). $\mathrm{Ce}$ résultat peut paraître surprenant puisque l'ACS se présente comme une aubaine pour les personnes ayant déjà engagé des dépenses pour l'achat d'un contrat de complémentaire santé $^{16}$ dont on aurait pu attendre un recours massif, augmenté d'autant par l'augmentation $\mathrm{du}$ « chèque santé ».

Ces résultats posent d'emblée la question du nombre de courriers qui sont réellement parvenus aux assurés. Celui-ci n'est pas connu précisément, mais il est probable que certaines adresses, pourtant transmises par la CPAM de Lille-Douai et la CAF de Lille, aient été erronées, et ce d'autant plus que la mobilité est étroitement associée à la précarité.

Ces résultats suggèrent également une imparfaite compréhension du dispositif et de l'information contenue dans les courriers par les bénéficiaires. Ceci rend sans doute difficile l'interprétation de la réponse des assurés à l'augmentation du chèque comme le simple reflet d'une sensibilité aux prix des contrats d'assurance indépendamment des coûts informationnels et administratifs. Mais cela illustre surtout la difficulté pour une CPAM de communiquer sur un dispositif, tant sur son existence que sur les démarches à entreprendre pour en bénéficier. Le faible succès de la campagne d'information par courrier et la complexité du dispositif de l'ACS appellent sans doute la mise en place d'une information directe, en face-à-face.

Cependant, nos résultats montrent également que, au sein du groupe traité 2 , seules $9 \%$ des personnes invitées ont effectivement participé à la réunion d'information et que l'invitation à

\footnotetext{
15. On peut se référer à Guthmuller et al. (2012) pour un traitement économétrique de la sélection.

16. Rappelons toutefois que les personnes couvertes dans le cadre d'un contrat collectif obtenu par le biais de l'employeur ne sont pas éligibles à l'ACS. Néanmoins, compte tenu des caractéristiques sociales de la population cible de l'ACS, ce critère d'inéligibilité ne peut expliquer à lui seul l'absence de recours massif à l'ACS des personnes initialement couvertes.
} 
s'informer dans les locaux de la CPAM décourage certains d'entreprendre des démarches. Ceci légitime certainement de recourir à des institutions tiers (association, mutuelles, assistantes sociales ...) pour diffuser l'information (Chauveaud et Warin, 2009).

Par ailleurs, parmi les dossiers retournés à la CPAM de Lille-Douai, seuls $55 \%$ ont au final donné lieu à une notification effective de l'ACS, conduisant seulement $9 \%$ des personnes incluses dans l'expérimentation à obtenir l'ACS. Ce taux de refus des dossiers est considérable pour une population présélectionnée au regard de ses ressources et des prestations sociales perçues par la CAF. Il s'agit d'un élément essentiel à prendre en compte. Le coût des démarches à entreprendre pour les personnes potentiellement éligibles est sans doute très sensiblement renforcé par la faible probabilité de succès. Ceci est commun à toutes les prestations sous conditions de ressources mais particulièrement renforcé dans le cas de l'ACS, compte tenu de la complexité des critères d'éligibilité et de l'étroitesse de la cible de niveaux de vie.

Ces intuitions sont confirmées par l'analyse qualitative qui a été menée pour recueillir les besoins et les attentes des personnes ciblées par l'ACS lors de l'observation des réunions d'une part et d'entretiens approfondis avec des bénéficiaires potentiels dont certains étaient venus à la réunion d'autre part (cf. encadré 6 ; pour une présentation détaillée voir Wittwer et al., 2010).

\section{Les enseignements de l'expérimentation}

L e choix d'expérimenter un dispositif réaliste, mis en ouvre in vivo, permet d'éclairer de façon robuste et pragmatique les politiques visant à améliorer le recours à l'ACS. En raison du coût élevé et des difficultés de mise en œuvre d'une expérimentation sociale, celleci n'a été conduite que sur un seul site et auprès de 4209 assurés. La population étudiée n'est donc représentative que de la population potentiellement éligible à l'ACS à Lille, qui présente certainement des caractéristiques particulières. De même, l'efficacité des modifications du dispositif ACS proposées est dépendante de l'institution qui l'a conduite et des relations qu'elle entretient avec ses assurés sociaux, surtout les plus modestes. Rien ne dit donc qu'une même expérimentation sur une population similaire aurait eu les mêmes effets dans une autre CPAM. Enfin, comme toute expérimentation, celle-ci est limitée dans le temps. Elle ne permet donc pas d'observer les éventuelles répercussions à long terme de la majoration du chèque santé en complémentarité avec la lente diffusion de l'information.

Cette expérimentation montre de manière robuste que la majoration du montant du " chèque santé " améliore légèrement le taux de recours et l'ACS et permet de mieux cibler les populations effectivement éligibles. Cela semble donc supposer un effet positif sur le recours à l'ACS de l'augmentation du montant légal de l'aide mise en place au $1^{\text {er }}$ janvier 2010 pour les personnes éligibles âgées de 50 ans et plus (cf. tableau, encadré 1). Néanmoins, cette majoration étant d'un montant plus faible que celui proposé dans le cadre de cette expérimentation, on ne peut s'attendre qu'à un effet d'une ampleur très modeste. L'absence de différence de réaction au traitement entre les populations initialement couvertes et non couvertes suggère que le coût de la complémentaire santé n'est pas la seule cause du non recours. L'incitation pécuniaire n'est pas le seul levier d'action possible. L'accès à l'information et la simplification des démarches sont des voies à encourager. Néanmoins, l'expérimentation montre la difficulté de toucher la cible par une campagne d'information par courrier, telle que celle mise en œuvre au niveau national en 2008-2009, et le caractère contre-productif de l'invitation à une réunion d'information. Elle suggère enfin que l'incertitude pesant sur l'éligibilité est certainement un facteur aggravant du non-recours à l'ACS. A ce titre, l'élargissement de la cible au $1^{\text {er }}$ janvier 2011 (cf. encadré 1) peut constituer une première piste pour favoriser le recours à ce dispositif. Les politiques testées ici ont cependant un effet globalement modeste qui ne permettra probablement pas de répondre à la question du non-recours à l'ACS. 
Arnould M-L. et Vidal G. (2008), « Typologie des contrats les plus souscrits auprès des complémentaires en 2006 », Études et Résultats, n 663.

Auerbach D. et Ohri S. (2006), «Price and Demand for Non-Group Health Insurance "), Inquiry, vol. 43, $\mathrm{n}^{\circ} 2$, pp. 122-134.

Boisguérin B., Després C., Dourgnon P., Fantin R. et Legal R. (2010), « Étudier l'accès aux soins des assurés CMU-C, une approche par le renoncement aux soins », dans Santé, soins et protection sociale en 2008, rapport IRDES, pp. 31-40.

Chauveaud C. et Warin P. (2009), « Favoriser l'accès aux soins des bénéficiaires de minima sociaux. Expliquer la CMU-C et l'ACS aux populations potentiellement éligibles », Odenore, collection Étude $\mathrm{n}^{\circ} 32$.

Commission des Comptes de la Sécurité sociale du 26 septembre 2006 (2006), « Présentation du PLFSS $2007 »$.

Desprès C. (2010), «La Couverture maladie universelle, une légitimité contestée : analyse des attitudes de médecins et dentistes à l'égard de ses bénéficiaires », Pratiques et Organisation des soins, vol. 41, $\mathrm{n}^{\circ}$ 1, pp. 33-43.

Elbaum M. (2008), « Participation financière des patients et équilibre de l'Assurance maladie », Lettre de l'OFCE, n' 301.

Fonds CMU (2008), «L'aide à la complémentaire santé en 2007 », Rapport du Fonds CMU au Gouvernement sur l'évolution du prix et du contenu des contrats ayant ouvert droit à l'aide complémentaire santé en 2007 (en application de l'article L.863-5 du Code de la Sécurité Sociale), juin 2008.

Fonds CMU (2009), Rapport d'activité $2008 d u$ Fonds CMU, 13 mai 2009. Téléchargeable sur le site : http ://www.cmu.fr/userdocs/Rapport \%202008.pdf.

Fonds CMU (2010), « L'aide à la complémentaire santé en 2009 », Rapport du Fonds CMU au Gouvernement sur l'évolution du prix et $d u$ contenu des contrats ayant ouvert droit à l'aide complémentaire santé en 2009 (en application de l'article L.863-5 du Code de la Sécurité Sociale), juin 2010.

Fonds CMU (2012a), Rapport d'activité $2011 d u$ Fonds CMU, 31 mai 2012. Téléchargeable sur le site : http ://www.cmu.fr/userdocs/Rapport_activite_2011.pdf.

Fonds CMU (2012b), « Bénéficiaires de l’ACS. Séries mensuelles des attestations délivrées et trimestrielles des attestations utilisées ", 9 juillet 2012. Téléchargeable sur le site: http ://www. cmu.fr/userdocs/Nb_benef_ACS_07_2012.pdf

Grignon M. et Kambia-Chopin B. (2009), "Income and the Demand for Complementary Health Insurance in France », Document de travail Irdes, $\mathrm{n}^{\circ} \mathrm{DT} 24$.

Guthmuller S., Jusot F. et Wittwer J. (2012), « Improving Take-up of Health Insurance Program: Evidence from a Social Experiment in France », Cahiers de la Chaire Santé n 11, Juin 2012.

Haut conseil à l'avenir de l'Assurance maladie (2005), Rapport 2005 du Haut conseil pour l'avenir de l'Assurance maladie adopté le vendredi 8 juillet 2005 .

Haut conseil à l'avenir de l'Assurance maladie (2006), Rapport 2006 du Haut conseil pour l'avenir de l'Assurance maladie, juillet 2006.

Haut conseil à l'avenir de l'Assurance maladie (2007), Rapport 2007 du Haut conseil pour l'avenir de l'Assurance maladie, juillet 2007.

Hernanz V., Malherbet F. et Pellizzari M. (2004), « Take-up of welfare benefits in OECD countries: review of the evidence ", Document de travail de l'OECD sur les affaires sociales, l'emploi et les migrations, $\mathrm{n}^{\circ} 17$.

Jusot F. et Wittwer J. (2009), « L'accès financier aux soins en France : bilan et perspective », Regards croisés sur l'économie, vol. 5, $\mathrm{n}^{\circ} 1$, pp. 102-109.

Jusot F., Perraudin C. et Wittwer J. (2012), «L'accessibilité financière à la complémentaire santé en France : les résultats de l'enquête Budget de Famille $2006 »$, Économie et Statistique, $\mathrm{n}^{\circ} 450$, pp. $29-46$.

Kambia-Chopin B., Perronnin M., Pierre A. et Rochereau T. (2008), « La complémentaire santé en France en 2006 : un accès qui reste inégalitaire. Résultats de l'enquête Santé protection sociale 2006 (ESPS 2006) », Questions d'économie de la santé, $\mathrm{n}^{\circ} 132$. 
Paita M. et Weill A. (2009), « Les affections de longue durée au 31 décembre 2008 », Points de repère $n^{\circ} 27$, Cnamts.

Perronnin M., Pierre A. et Rochereau T. (2011), « La complémentaire santé en France en 2008 : une large diffusion mais des inégalités d'accès ", Questions d'économie de la santé, $\mathrm{n}^{\circ} 161$.

Revil H. (2008), «Identifier des populations en non-recours aux dispositifs de l'Assurance maladie : proposition de méthode », Recherches et Prévisions, ${ }^{\circ}$ 93, pp. 102-109.

Roy A.D. (1951), «Some Thoughts on the Distribution of Earnings », Oxford Economic Papers, New Series, vol. 3, n 2, pp. 135-146.
Rubin D.B. (1974), « Estimating causal effects of treatments in randomized and nonrandomized studies ", Journal of Educational Psychology, vol. $66, \mathrm{n}^{\circ} 5$, pp. 688-701.

Thomas, K. (1995), «Are subsidies enough to encourage the uninsured to purchase health insurance? An analysis of underlying behavior ", Inquiry, vol. 31, $\mathrm{n}^{\circ} 4$, pp. 415-424.

Wittwer J., Jusot F., Guthmuller S., Desprès C. et Renaud T. (2010), « Le recours à l'Aide complémentaire santé à Lille : résultats d'une expérimentation sociale ", Rapport final dans le cadre de l'appel à projet d'expérimentations sociales 2008, Haut commissariat aux Solidarités actives contre la pauvreté. 
Le tableau présente les effets marginaux de la probabilité de retourner un dossier complété estimés à partir d'un modèle Probit. Dans les modèles 1 et 3 , seule l'appartenance au groupe est modélisée. Dans les modèles 3 et 4 , l'effet du groupe traité 2 est décomposé en une indicatrice "avoir assisté à la réunion" et une autre décrivant l'impact lorsque l'assuré n'y a pas assisté. Dans les modèles 2 et 4 , nous contrôlons également des caractéristiques individuelles des assurés avant le début de l'expérimentation.

Tableau

Effets marginaux de la probabilité de retourner un dossier complété

\begin{tabular}{|c|c|c|c|c|}
\hline & Modèle 1 & Modèle 2 & Modèle 3 & Modèle 4 \\
\hline Probabilité prédite & 0,166 & 0,165 & 0,151 & 0,150 \\
\hline \multicolumn{5}{|l|}{ Variables explicatives } \\
\hline $\begin{array}{l}\text { Groupe } \\
\text { Témoin } \\
\text { Traité } 1 \\
\text { Traité } 2 \\
\text { Traité } 2 \times \text { réunion } \\
\text { Traité } 2 \times \text { sans réunion }\end{array}$ & $\begin{array}{l}-0,025^{\star \star} \\
\text { Réf. } \\
-0,030^{\star \star}\end{array}$ & $\begin{array}{l}-0,024^{\star \star} \\
\text { Réf. } \\
-0,028^{\star \star}\end{array}$ & $\begin{array}{c}-0,025^{\star \star} \\
\text { Réf. } \\
- \\
0,088^{\star \star} \\
-0,042^{\star \star \star} \\
\end{array}$ & $\begin{array}{c}-0,024^{\star \star} \\
\text { Réf. } \\
- \\
0,068^{\star \star} \\
-0,039^{\star \star \star}\end{array}$ \\
\hline $\begin{array}{l}\text { Age } \\
\text { Age }^{2}\end{array}$ & & $\begin{array}{c}0,012^{\star \star \star} \\
-0,0001^{\star \star \star}\end{array}$ & & $\begin{array}{c}0,012^{\star \star \star} \\
-0,0001^{\star \star \star}\end{array}$ \\
\hline $\begin{array}{l}\text { Sexe } \\
\text { Femme } \\
\text { Homme }\end{array}$ & & $\begin{array}{c}-0,024^{\star \star} \\
\text { Réf. }\end{array}$ & & $\begin{array}{c}-0,024^{\star \star} \\
\text { Réf. }\end{array}$ \\
\hline $\begin{array}{l}\text { Régime de l'assuré } \\
\text { Actif } \\
\text { Retraité } \\
\text { Invalide }\end{array}$ & & $\begin{array}{c}\text { Réf. } \\
0,157^{\star \star \star} \\
0,159^{\star \star \star}\end{array}$ & & $\begin{array}{l}\text { Réf. } \\
0,158^{\star \star \star} \\
0,161^{\star \star \star}\end{array}$ \\
\hline Ayant droit de moins de 3 ans & & 0,030 & & 0,030 \\
\hline Bénéficiaire de la CMUC en 2007 & & 0,029 & & 0,029 \\
\hline Couvert par une CS en 2008 & & 0,016 & & 0,018 \\
\hline En ALD en 2008 & & $-0,006$ & & $-0,007$ \\
\hline $\begin{array}{l}\text { Dépenses de soins ambulatoires en } 2008 \\
<200 € \\
200 €-699 € \\
700 €-1999 € \\
>=2000 €\end{array}$ & & $\begin{array}{c}-0,054^{\star \star \star} \\
-0,007 \\
0,007 \\
\text { Réf. }\end{array}$ & & $\begin{array}{c}-0,054^{\star * \star} \\
-0,006 \\
0,006 \\
\text { Réf. }\end{array}$ \\
\hline $\begin{array}{l}\text { Qualité du modèle } \\
\text { AIC (AIC constante seule = 3793,214) } \\
\text { Paires concordantes (en \%) } \\
\mathrm{R}^{2} \text { ajusté }\end{array}$ & $\begin{array}{c}3791,651 \\
83,4 \\
0,001\end{array}$ & $\begin{array}{c}3586,32 \\
83,4 \\
0,054\end{array}$ & $\begin{array}{c}3779,489 \\
83,4 \\
0,003\end{array}$ & $\begin{array}{c}3577,365 \\
83,4 \\
0,056\end{array}$ \\
\hline Nombre d'observations & 4209 & 4209 & 4209 & 4209 \\
\hline
\end{tabular}

Lecture : dans le modèle 1, appartenir au groupe témoin diminue la probabilité de retourner un dossier complété de 2,5 points de pourcentage comparativement à la probabilité du groupe traité 1.

Champ : ensemble de la population dans le champ d'expérimentation.

Source : données et calculs des auteurs. 